\title{
Overexpression of Human Mutant PANK2 Proteins Affects Development and Motor Behavior of Zebrafish Embryos
}

\author{
D. Khatri ${ }^{1}$ - D. Zizioli ${ }^{1}$ A. Trivedi $^{1}$ - G. Borsani ${ }^{1}$ - E. Monti ${ }^{1}$ - D. Finazzi ${ }^{1,2}$ (B)
}

Received: 2 June 2018 / Accepted: 17 August 2018 / Published online: 23 August 2018

(c) Springer Science+Business Media, LLC, part of Springer Nature 2018

\begin{abstract}
Pantothenate Kinase-Associated Neurodegeneration (PKAN) is a genetic and early-onset neurodegenerative disorder characterized by iron accumulation in the basal ganglia. It is due to mutations in Pantothenate Kinase 2 (PANK2), an enzyme that catalyzes the phosphorylation of vitamin B5, first and essential step in coenzyme A (CoA) biosynthesis. Most likely, an unbalance of the neuronal levels of this important cofactor represents the initial trigger of the neurodegenerative process, yet a complete understanding of the connection between PANK2 malfunctioning and neuronal death is lacking. Most PKAN patients carry mutations in both alleles and a loss of function mechanism is proposed to explain the pathology. When PANK2 mutants were analyzed for stability, dimerization capacity, and enzymatic activity in vitro, many of them showed properties like the wild-type form. To further explore this aspect, we overexpressed the wild-type protein, two mutant forms with reduced kinase activity and two retaining the catalytic activity in zebrafish embryos and analyzed the morpho-functional consequences. While the wild-type protein had no effects, all mutant proteins generated phenotypes that partially resembled those observed in pank2 and coasy morphants and were rescued by CoA and vitamin B5 supplementation. The overexpression of PANK2 mutant forms appears to be associated with perturbation in CoA availability, irrespective of their catalytic activity.
\end{abstract}

Keywords Pantothenate Kinase 2 (PANK2) · Pantothenate Kinase-Associated Neurodegeneration (PKAN) · Coenzyme A · Neurodegeneration $\cdot$ Zebrafish

\section{Introduction}

Mutations in PANK2 gene are associated with an autosomal recessive neurodegenerative disorder named Pantothenate Kinase-Associated Neurodegeneration (PKAN, phenotype MIM \#234200; gene MIM 606157). It is a very rare disease characterized by iron accumulation in the basal ganglia, particularly in the Globus Pallidus, and hence included in the novel nosographic category of neurodegeneration with brain iron accumulation (NBIA) (Hayflick 2003). A classic and an

Electronic supplementary material The online version of this article (https://doi.org/10.1007/s12017-018-8508-8) contains supplementary material, which is available to authorized users.

D. Zizioli

daniela.zizioli@unibs.it

1 Department of Molecular and Translational Medicine, University of Brescia, viale Europa 11, 25123 Brescia, Italy

2 Clinical Chemistry Laboratory, Department of Diagnostic, ASST Spedali Civili di Brescia, 25123 Brescia, Italy atypical form can be differentiated; the first one is characterized by early onset, and more rapid progression with death often occurring within the 2 nd or 3 rd decade of life. The atypical form is less common and with more heterogeneous symptoms. The progression is slower with patients living into their fifties (Gregory et al. 2009).

$P A N K 2$ gene codes for an enzyme catalyzing the first step in coenzyme $\mathrm{A}(\mathrm{CoA})$ biosynthesis that is the phosphorylation of pantothenic acid or vitamin B5 to phosphopantothenate. This is the rate-controlling step in the process (Leonardi et al. 2005), and hence, PANK expression levels significantly contribute to the regulation of cellular CoA content (Rock et al. 2000; Zhang et al. 2007). The activities of three downstream enzymes, phosphopantothenoylcysteine synthetase, phosphopantothenoylcysteine decarboxylase, and COASY, catalyze the condensation with cysteine and ATP and the final phosphorylation of dephospho-CoA, leading to the final production of CoA. In humans, there are at least two other genes coding for active PANK proteins, namely $P A N K 1$ and $P A N K 3$. Another fourth paralog, PANK4, showed no catalytic activity when expressed in mammalian cells (Zhang 
et al. 2007) and partial rescue efficiency in a Drosophila strain $\left(f b l^{l}\right)$ carrying a hypomorphic allele of the sole pantothenate kinase gene (fumble) (Wu et al. 2009). PANK1, 2, and 3 all share a common C-terminal catalytic domain, but differ at the $\mathrm{N}$-terminal domain, which is involved in determining the specific features of each enzyme (Leonardi et al. 2007b). Notably, the N-terminal portion of PANK2 contains nuclear localization and export signals together with a mitochondrial targeting signal and the protein can be found in the nucleus and in the mitochondrial inter-membrane space (Leonardi et al. 2007b; Brunetti et al. 2012; Alfonso-Pecchio et al. 2012). PANK1a localizes in the nucleus, while PANK1b is found in the cytosol, partially associated with recycling endosomes and clathrin-coated vesicles. PANK3 has a very short $\mathrm{N}$-terminal domain and resides in the cytosol (Alfonso-Pecchio et al. 2012). These isoforms present also a different tissue distribution: PANK1 is expressed at higher level in liver, heart, and kidney, PANK2 and PANK3 are ubiquitously expressed, with the first one being more abundant in the brain and the latter in the liver (Leonardi et al. 2007b). All PANK biochemical activities are controlled by feedback signals by non-esterified and esterified CoA, but each isoform presents different $\mathrm{IC}_{50}$, with PANK2 showing the highest sensitivity (Zhang et al. 2006; Leonardi et al. 2007a). These results suggest that the different PANK isoforms are not redundant enzymes and each contributes to the fine-tuning of $\mathrm{CoA}$ production according to the specific needs of different cells and tissues.

Loss of function of PANK2 is associated with PKAN, but a clear understanding of the molecular process leading to neuronal death is not available yet. The most likely hypothesis is that the genetic mutations would lead to a chronic decrease in CoA content in neurons, but direct evidence in human tissues are not available. The identification of patients with a similar clinical phenotype and carrying mutation in COASY (Dusi et al. 2014), the enzyme involved in the final step of CoA biosynthesis clearly supports this theory. Further robust data come from studies performed in Drosophila (Rana et al. 2010; Srinivasan et al. 2015), zebrafish (Zizioli et al. 2016), mice (Brunetti et al. 2014), and patient-derived neurons (Orellana et al. 2016). Other important aspects that have been partially investigated are the biochemical consequences of the point mutations on PANK2 functioning and the genotype-phenotype relationship. Several types of sequence variations have been documented in PKAN patients. They are distributed along the entire PANK2 sequence without any hotspot. Most mutations are single-nucleotide changes or indels resulting in missense or frameshift changes and premature stop codons. The in vitro analyses of the effects of the most common NBIA-associated missense mutations on protein stability, mitochondrial targeting, sensitivity to acetyl-CoA inhibition and enzymatic activity revealed interesting and somewhat surprising differences (Kotzbauer et al. 2005; Zhang et al. 2006). No mutation was found to alter the processing, the final protein localization, and the feedback regulation, while the p.G521R amino acid substitution (c.1561G $>$ A) resulted in a highly unstable and short-lived protein. Some mutations (p.G219V, p.S471N, p.G521R, p.N500I) revealed a dramatic loss $(\geq 85 \%)$ of the catalytic activity, but many others (p.T528M, p.T234A, p.N404I, p.R286C, p.T327I) showed normal or even increased kinase activity. Interestingly, the p.G521R and p.T528M were also analyzed for rescue efficiency in $f b l^{1}$ flies (Wu et al. 2009). The first mutant had very low kinase activity when expressed in Drosophila and was unable to recover any of the defects observed in $f b l^{1}$ larvae; the latter showed about $70 \%$ kinase activity when compared to the normal control and partially rescued most of the abnormalities, except for male sterility. Altogether these results suggest possible differences between in vivo and in vitro assays in monitoring PANK2 functioning and indicate that the absence of catalytic activity may be not the unique cause of PKAN. Nonetheless, the hypothesis of other yet unknown PANK2 functions seems to be less plausible, given the in vitro and in vivo rescue efficiency of molecules (pantethine, 4-phosphopantetheine, $\mathrm{CoA}$ ) that bypass PANK2 activity in the CoA biosynthetic process (Siudeja et al. 2011; Brunetti et al. 2014; Srinivasan et al. 2015; Orellana et al. 2016; Zizioli et al. 2016; Khatri et al. 2016). Hartig et al. (2006) conducted a systematic analysis of the genotypic and phenotypic spectrum in 48 NBIA patients carrying PANK2 mutations. An activity score for each PANK2 protein type was assigned based on previous biochemical data (Kotzbauer et al. 2005) and on the evolutionary conservation of the amino acid affected by each specific mutation. They observed a linear correlation between the activity score and the age of onset, while the rate of progression of the disease, measured as the time interval from onset to loss of ambulation, appeared to be independent from the genotype.

We recently showed that zebrafish embryos represent a valuable experimental setting to investigate the effects of defects in CoA biosynthesis (Khatri et al. 2016; Zizioli et al. 2016), providing information about different cells and tissues sensitivity to pank 2 and coasy downregulation. Zebrafish expresses four different Panks that are considered orthologues of human PANK1a, PANK1b, PANK2, and PANK4, according to sequence homology and synteny conservation. We decided to investigate PANK2 mutant forms by overexpressing them in zebrafish embryos. This approach is very useful to investigate mutations associated with a dominant negative effect, but we reasoned that, in the case of zebrafish embryos, it could be valuable also for loss of function mutants that still retain a partial activity. Two features should contribute to make our approach feasible: (i) pank2 is a maternal gene in zebrafish (Zizioli et al. 2016); its expression drops to lower levels in the 
first stages of development and then progressively raises to higher levels since 24-h post-fertilization (hpf). So, it should be possible to overcome the amount of endogenous protein by microinjection of exogenous mRNA; (ii) zebrafish embryos rely on the inherited pool of vitamin B5 and CoA for their development and do not find these molecules in the environment. It is then possible that a large excess of mutant protein suppresses the activity of the endogenous form by competing for the limiting amount of vitamin B5 available in embryos. We injected embryos with $50 \mathrm{pg}$ of human wild-type (WT) and mutant PANK2 mRNAs and verified the consequences at morphological and behavioral levels. All the embryos overexpressing mutant proteins resulted in aberrant phenotypes, with degrees of severity correlating to the type of injected mutant mRNA. CoA or vitamin B5 addition to the water rescued all of them, thus confirming the direct relationship between PANK2 activity and CoA homeostasis.

\section{Materials and Methods}

\section{Fish Breeding, Embryo Collection, and Treatments}

Wild-type zebrafish $\mathrm{AB}$ strain and the transgenic lines $\operatorname{Tg}$ (neurod1:EGFP) and $\operatorname{Tg}($ flila:EGFP) were used according to EU Directive 2010/63/EU for animal experiments. Zebrafish were maintained and used by following the protocols approved by the Local Committee (Organismo per il benessere animale - Committee for animal health). They were all kept in tanks containing $3 \mathrm{~L}$ of water at $28^{\circ} \mathrm{C}$ with 14-h light and 10-h dark cycle. Breeding of adult zebrafish was carried out by natural crosses and embryos were collected; they were staged according to established protocols (Kimmel et al. 1995). Collected embryos were grown at $28{ }^{\circ} \mathrm{C}$ in fish water containing $0.1 \mathrm{~g} / \mathrm{L}$ Instant Ocean Sea Salts, $0.1 \mathrm{~g} / \mathrm{L}$ sodium bicarbonate, $0.19 \mathrm{~g} / \mathrm{L}$ calcium sulfate, $0.2 \mathrm{mg} / \mathrm{L}$ methylene blue in sterile water, until the desired developmental stage was reached. To examine post-gastrulation stages, fish water was added by $0.0045 \%$ PTU (1-phenil-2-thiourea, Sigma) solution. Embryos were dechorionated by hand using sharpened forceps and fixed in $4 \%$ paraformaldehyde $1 \times$ PBS overnight at $4{ }^{\circ} \mathrm{C}$, dehydrated through sequential washes in $25 \%, 50 \%, 75 \%$ methanol/PBS, $100 \%$ methanol and stored at least overnight at $-20{ }^{\circ} \mathrm{C}$. The oldest age at which the zebrafish embryos were sacrificed is $72 \mathrm{hpf}$. Effects of CoA, vitamin B5, and ascorbic acid (Sigma) upon embryos development were examined after addition of each specific drug directly in the fish-water at $4 \mathrm{hpf}$ (blastula stage); dose curve experiments were performed as described previously (Zizioli et al. 2016).

\section{Whole-Mount in Situ Hybridization}

Whole-mount in situ hybridization (WISH) was performed according to a standard method (Thisse and Thisse 2008). In Brief, embryos were collected, dechorionated, and incubated at $28{ }^{\circ} \mathrm{C}$ at different stages. Embryos were fixed overnight in $4 \%$ paraformaldehyde (PFA) at $4{ }^{\circ} \mathrm{C}$, dehydrated through an ascending methanol series and stored at $-20{ }^{\circ} \mathrm{C}$. After treatment with proteinase $\mathrm{K}(10 \mu \mathrm{g} / \mathrm{mL}$, Roche $)$, the embryos were hybridized overnight at $68^{\circ} \mathrm{C}$ with DIG-labeled antisense or sense RNA probes (400 ng). Embryos were washed with ascending scale of Hybe Wash/PBS and SSC/PBS and then incubated with anti-DIG antibody conjugated with alkaline phosphatase overnight at $4{ }^{\circ} \mathrm{C}$. The staining was performed with NBT/BCIP (blue staining solution, Roche) alkaline phosphatase substrates. When different types of probes or fish had to be compared, all incubations were carried out at the same time, at the same probe concentration and, when possible, with the same reagents and solutions. WISH images were taken with a Leica MZ16F stereomicroscope equipped with DFC 480 digital camera and LAS Leica Imaging software (Leica).

\section{In Vitro Synthesis of PANK2 WT and Mutant mRNAs}

Recombinant plasmids (pcDNA 3.1 myc-his tag) containing the coding region of human $P A N K 2$, both WT and mutant forms (G219V, S471N, T234A, and T528M) were a generous gift from Prof. Kotzbauer (University of Pennsylvania). $3 \mu \mathrm{g}$ of each plasmid was digested with $X b a \mathrm{I}$. The insert containing the specific PANK2 cDNA was gel-purified and transcribed with T7 RNA polymerase using the mMESSAGE mMACHINE T7 in vitro transcription kit (Ambion) according to the manufacturer's instructions.

\section{Microinjections}

In vitro synthesized mRNAs were injected together with the dye tracer rhodamine dextran in $1 \times$ Danieau buffer $(\mathrm{pH} 7.6)$ into 1- to 2-cell stage embryos, according to an established protocol (Nasevicius and Ekker 2000). After microinjection, embryos were incubated in fish water supplemented with $0.003 \%$ PTU at $28{ }^{\circ} \mathrm{C}$ to prevent pigmentation processes. Embryo development was evaluated at 24 and $48 \mathrm{hpf}$.

\section{Microscopy}

The images of embryos were obtained with two different microscopes: (1) a compound Leica DMR microscope, equipped with Nomarski optics and a DC500 Leica digital camera; (2) Zeiss Axio Zoom V16 equipped with Zeiss Axiocam 503 color. Fluorescence of transgenic lines was visualized using a Leica M165FC dissecting microscope. 
For in vivo analyses, embryos and larvae were anesthetized with tricaine $0.16 \mathrm{mg} / \mathrm{mL}$ and mounted in $1 \%$ low melting agarose gel.

\section{Acridine Orange Staining}

For acridine orange (AO) staining, embryos at $48 \mathrm{hpf}$ were in vivo dechorionated and incubated for $30 \mathrm{~min}$ in fish water containing $\mathrm{AO}$ at $10 \mathrm{mg} / \mathrm{L}$. Embryos were then rinsed three times in fish water, anesthetized with tricaine, and quickly imaged under a dissecting microscope with green fluorescent filter.

\section{RNA Extraction and Real-Time RT-PCR}

Total RNA was extracted from 50 embryos, using TRIReagent (Sigma) according to manufacturer's protocol. RNA was quantified using the My Spect spectrophotometer (Biomed) and controlled by electrophoretic separation on a $1 \%$ TAE-agarose gel. $1 \mu \mathrm{g}$ of total RNA was retrotranscribed to cDNA using Im-Prom Reverse Transcriptase (Promega) and oligo (dT) primers. Primers were designed by the Real-Time PCR Tool from IDT with the following sequences: zebrafish pank2 (5'-TCCATGAAGTTGCTC GCGTA-3', 5'-CAGCTCAGGATGGGTTTGGT-3'), human PANK2 (5'-GGATCGACTGGGCTCTTACA-3' and 5'-TTG GAGGTCAGGTACTTCCG-3'), and zebrafish actin beta 1 (5'-CGAGCAGGAGATGGGAACC-3' and 5'-CAACGG AAACGCTCATTGC-3'). Real-time PCR was performed using the PCR MAX ECO48 system, in a $10 \mu \mathrm{L}$ volume, with $0.5 \mu \mathrm{M}$ of each primer, $5 \mu \mathrm{L}$ of Syber Green Master Mix (Biorad) and $20 \mathrm{ng}$ of cDNA. The amplification profile consisted of a denaturation program $\left(95^{\circ} \mathrm{C}\right.$ for $\left.1 \mathrm{~min}\right), 40$ cycles with two steps $\left(95^{\circ} \mathrm{C}\right.$ for $15 \mathrm{~s}$ and $60{ }^{\circ} \mathrm{C}$ for $\left.30 \mathrm{~s}\right)$ followed by a melting cycle. Each reaction was performed in triplicate. Relative levels of expression were calculated by the $\Delta \Delta \mathrm{CT}$ method, as previously described (Gatta et al. 2009).

\section{Immunoblotting}

30 embryos for each condition were manually dechorionated. Proteins were extracted by homogenizing embryos in buffer (200 mM Tris/ $\mathrm{HCl}, 100 \mathrm{mM} \mathrm{NaCl}, 1 \mathrm{mM}$ EDTA, $0.5 \% \mathrm{NP}-40,10 \%$ Glycerol, $1 \mathrm{mM} \mathrm{NaFl}$ and sodium orthovanadate) on ice and quantified by a standard bicinchoninic acid method. $50 \mu \mathrm{g}$ of total protein extract was then separated on $10 \%$ polyacrylamide gels, transferred on to membranes (GE Amersham ${ }^{\mathrm{TM}}$ Hybond $^{\mathrm{TM}} \mathrm{P} 0.45$ PVDF). Membranes were cut at the level of the molecular weight of interest, blocked with $2 \%$ skim milk in TBST, incubated with the primary antibody diluted in $2 \%$ skim milk in TBST (PANK2, 1:1000, Origene \#TA501321) and then with the anti-mouse IgG secondary antibody also prepared in $2 \%$ skim milk (1:2000). ECL Western blotting detection reagent (Pierce) was used for detection. Images were acquired with Li-Cor Odissey image station, and band intensity quantified by ImageJ software without any modification of the original data. Original images were partially cropped to fit to the final figure. A polyclonal antibody against human PANK1b (generous gift by dr. Suzanne Jackoski, St. Jude Children's Research Hospital, Memphis, TN, USA) was also tested for the capacity to recognize zebrafish Pank2.

\section{Behavioral Assays}

Zebrafish embryos were injected with different mRNAs and incubated in fish water non-injected embryos were used as negative controls. Embryos were kept at $28^{\circ} \mathrm{C}$ until reaching the correct stage of development to carry out the test. At 21 hpf, 40 embryos for each mRNA were observed for head-tail coil spontaneous contractions for 1 min under Leica MZ16F stereomicroscope (Leica Microsystems Ltd.). The total number of head-tail coil movements of three independent experiments was recorded for each embryo, the average was calculated and a box diagram was plotted for treated and control embryos.

At $48 \mathrm{hpf}, 40$ embryos for each type were subjected to touch-and-response test, following the protocol by (Goody et al. 2012), with slight modification. A motility wheel, consisting in four concentric circles of respectively 5, 10, 15, $20 \mathrm{~mm}$ diameter, was placed on the microscope stage and centered at the bottom of a 60-mm Petri dish containing fish water. A single embryo was transferred in the middle of the concentric circles and when it was stationary in the center of the plate the test could start. Each embryo was gently touched at tail with an embryo poker and the distance it swam in the predetermined concentric circles was recorded. If the embryo did not completely exit the first designated circle, it was repositioned in the center of the plate and the test was repeated. If the embryo could not cross the first circle after multiple attempts ( 5 attempts), it was determined as incapable of exiting the circle. Once data of the distance swam by all 40 embryos of each group were obtained, the percentage of embryos crossing each predetermined concentric circle were calculated.

\section{Statistical Analysis}

The data are reported as representative of two or more experiments with similar results, depending on the number of embryos analyzed in each experiment. Statistically significant differences between different types of groups were calculated by one-way ANOVA analyses $(F=672.9, \mathrm{~d} f=5$, $* p<0.05 ; * * p<0.005 ; * * * p<0.0001)$ or $T$-Student Test (d $\left.f=78,{ }^{\#} p<0.05\right)$. 


\section{Results}

\section{Overexpression of Human PANK2 mRNA in Zebrafish Embryos}

To investigate the in vivo effects of mutations in PANK2 gene, we decided to overexpress human WT and mutant $P A N K 2$ mRNAs in zebrafish embryos. Given the limited amount of vitamin B5 available in fish embryos, a large excess of mutant protein could compete for the substrate with the endogenous enzyme and hence limit its activity. A dose curve analysis was performed with human WT $P A N K 2$ mRNA to select the suitable amount of nucleic acid for microinjection (Online supplementary material, Figure S1, A). Non-injected embryos were used as control for the experiment. At $48 \mathrm{hpf}$, no specific morphologic alterations were observed at various doses, although the survival rate fell drastically with doses above $50 \mathrm{pg} / \mathrm{embryo}$. More than $90 \%(202 / 219)$ of embryos survived in the case of control, whereas $77 \%(174 / 225)$ and $72 \%(148 / 206)$ of embryos could survive when injected with 20 and 50 pg/embryos, respectively. More than $60 \%$ of embryos died at the dose of $100 \mathrm{pg} / \mathrm{embryo}$ or above. We selected the dose of $50 \mathrm{pg} /$ embryo of mRNA that also allowed the full recovery of the normal phenotype in embryos co-injected with $1 \mathrm{pg} / \mathrm{embryo}$ of a pank2 specific, splice inhibiting morpholino (Zizioli et al. 2016).

\section{Overexpression of Human PANK2 mRNAs in Zebrafish Embryos}

Four different human mutant $P A N K 2$ alleles were selected for injection based on published data (Wu et al. 2009; Zhang et al. 2006; Kotzbauer et al. 2005): the G219V and S471N forms (hereafter KOFF-Mt) that retain very low level of pantothenate kinase activity in vitro and the T234A and T528M (hereafter KON-Mt) that retain a normal in vitro enzymatic activity. We injected either WT or mutant mRNAs in zebrafish embryos at one cell stage at the dose of $50 \mathrm{pg} /$ embryo. Embryos were grown in fish water at $28{ }^{\circ} \mathrm{C}$ and observed at 48 hpf (Fig. 1). No morphological changes were observed in embryos receiving WT human PANK2 mRNA as compared to non-injected embryos. Fish expressing KOFFMt mRNAs showed modest alterations in the ventral part of the tail. Specifically, the trunk was reduced in thickness and curved, the yolk extension was smaller or absent (G219V 218/271 and S471, 256/270). Some embryos showed modest signs of perturbation in blood circulation (S471N, 93/270). On the other hand, when embryos were injected with the T234A PANK2 form, severe alterations were observed in the caudal plexus. A big hemorrhage was present in the caudal plexus with accumulation of blood. Probably due to the poor supply of blood flow to the tail tip, tissues were not properly developed and the tail region was comparatively thinner than that of control embryos (239/280). Yolk extension was absent or under developed in all these embryos (280/280). Similarly, embryos overexpressing the T528M PANK2 form showed similar, albeit less evident phenotypic changes (256/278). Most of the embryos were thinner, had a curved tail structure, and lacked the formation of the yolk extension in the tail region. Blood vessels were not properly developed (239/278).

To verify that PANK2 mRNAs were all efficiently injected, we performed a real-time RT-PCR on injected and non-injected embryos (Online supplementary material, Figure S1, B). Levels of endogenous zebrafish pank2 mRNA were measured together with those of the exogenous human PANK2 mRNA at different time points (12, 24 and $48 \mathrm{hpf})$. The levels of zebrafish pank $2 \mathrm{mRNA}$ increased with time, as expected from our previous analysis (Zizioli et al. 2016). All exogenous mRNAs were present at similar levels at each time point (not shown). The amount of exogenous mRNAs were about 4 and twofold higher than

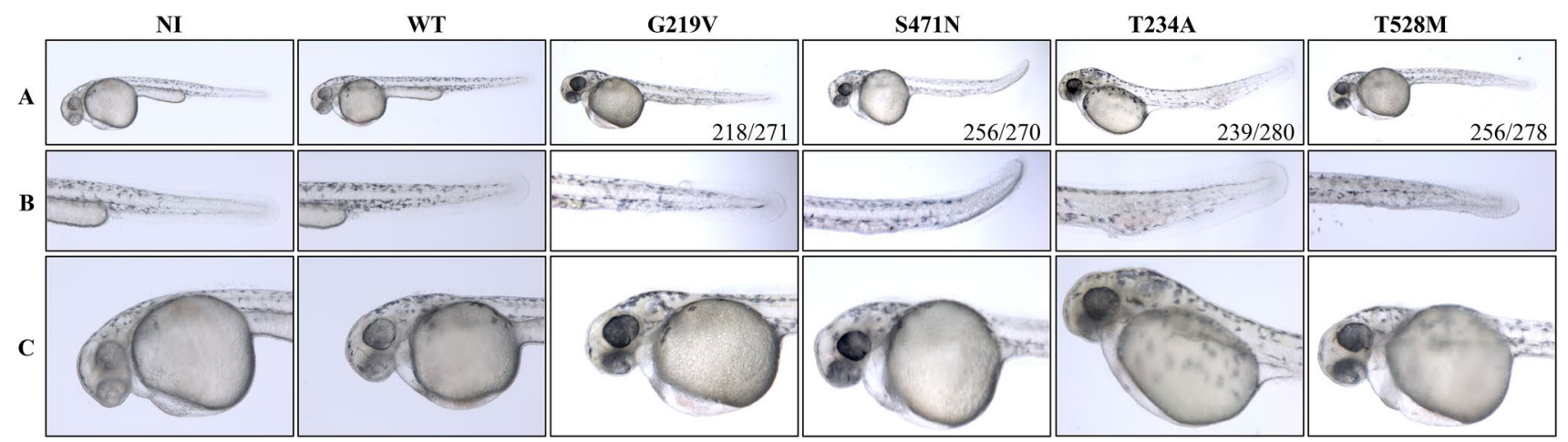

Fig. 1 Overexpression of WT and mutant PANK2 mRNAs in zebrafish embryos. Phenotypic assessment of embryos at $48 \mathrm{hpf}$ after injection of WT and mutant PANK2 mRNAs (50 pg/embryo).
Non-injected (NI) embryos were used as control. a Lateral views; $\mathbf{b}$ enlargements of the tail; $\mathbf{c}$ enlargements of the head. Representative images from one experiment out of a total of 4 with similar results 
that of the endogenous pank2 at 12 and $24 \mathrm{hpf}$, respectively. At $48 \mathrm{hpf}$, all exogenous mRNAs were drastically reduced, as expected because of the absence of the $5^{\prime}$ capping and the poly-adenilation tail. This confirms the efficiency of all microinjection and indicates that, in microinjected embryos, the amount of exogenous human PANK2 transcript exceeds the endogenous one during the first period of embryo development. To assess whether all exogenous mRNAs were similarly utilized in vivo we performed an immunoblot analysis of protein extracts at $12 \mathrm{hpf}$ (Online supplementary material, Figure S1, C). Samples were analyzed using a specific monoclonal antibody raised against the human protein. No band was detected in non-injected embryos, whereas we observed bands corresponding to immature and mature forms of PANK2 in all other cases. At 12 hpf, all the bands had similar intensity. To validate the analysis, we confirmed the linearity of the signal generated in the Western blotting in embryos injected with different amounts $(6.25,12.5$, 25 , and 50 pg/embryo) of WT PANK2 mRNA (Online supplementary material, Figure S2). We also tried to compare the level of expression of the endogenous and exogenous PANK2 protein in microinjected embryos, but none of the antibodies available to us (including a polyclonal antibody for human PANK1b) recognized the zebrafish protein in our hands.

To assess the specificity of the morphological sequelae associated with the injection of $50 \mathrm{pg} / \mathrm{embryo}$ of human PANK2 mRNAs, we repeated the experiment with a reduced dose (25 pg/embryo) of each mRNA (Online supplementary material, Figure S3). As expected, there were no morphological changes in the embryos injected with WT human PANK2 mRNA as compared to non-injected embryos. In embryos overexpressing the different mutant proteins, the phenotypes were characterized by features similar albeit less intense than those of previous experiments performed with the higher dose of $50 \mathrm{pg} / \mathrm{embryo}$. The embryos showed phenotypic changes in the ventral part of tail and improper formation of blood vessels and yolk (G219V 141/150, S471N 144/155, T234A, and T528M 150/150). As observed before, these alterations were modest in embryos overexpressing the KOFF-Mt forms and more evident in embryos injected with KON-Mt mRNAs.

\section{Co-expression of WT and Mutant PANK2 Allows Normal Development}

To exclude a possible association of the observed phenotype with the high amount of exogenous protein production induced in injected embryos, we tried to modulate the relative abundance of mutant and WT proteins by co-injecting different doses of WT PANK2 mRNA. We kept constant the total amount of injected-mRNA (50 pg/embryo), but mixed WT and mutant mRNA at the ratio of $1: 9,1: 1$ or 9:1. Irrespective of the type of mutant mRNA applied to embryos, we observed phenotypic changes (recapitulating those described above) only at the ratio of 1:9 (WT vs mut mRNA) whereas equal amount of WT and mutant mRNAs (1:1), or excessive amount of WT mRNA (9:1) resulted in a completely normal development (Fig. 2). These results suggest that (i) the described perturbations of development are not an artefact due to the excessive protein levels; (ii) the mutant proteins do not have a dominant negative effect.

\section{Mutant PANK2 Overexpression and Development of the Vascular Structure}

Embryos injected with KON-Mt forms of PANK2 showed severe perturbation in the development of the vascular structure, particularly at the caudal plexus and in the tail. To further explore this phenotype, injected embryos were grown till $36 \mathrm{hpf}$, then fixed with paraformaldehyde and analyzed by ISH with a cadherin 5 ( $c d h 5)$ specific RNA probe (Fig. 3, left panel). $C d h 5$ is a marker for vascular epithelium (Montero-Balaguer et al. 2009). ISH results evidenced that embryos injected with T234A mRNA had poor expression of $c d h-5$ in ISVs, which appeared reduced in size and length. The specific labeling in the region of the caudal plexus showed that this structure is severely perturbed, with

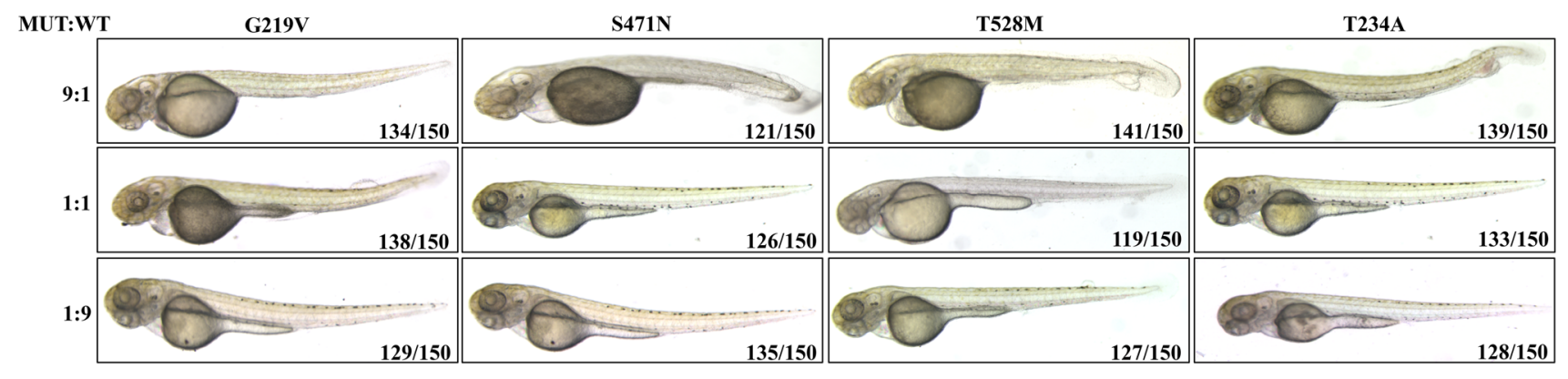

Fig. 2 Effects of co-expression of human WT and mutant PANK2 mRNAs in zebrafish embryos at $48 \mathrm{hpf}$. WT and mutant PANK2 mRNAs were injected at three different ratios of mutant to WT
mRNA (9:1, 1:1, 1:9). Morphological alteration was observed exclusively at the 9:1 ratio (MUT:WT). $(N=3)$ 
Fig. 3 Analysis of vascular development in embryos overexpressing WT and KONMt mRNAs. WISH analysis (Cdh5 probe) at $40 \mathrm{hpf}$ shows poor development of ISVs in embryos overexpressing T234A and T528M mutants. $\operatorname{Tg}($ Flila:EGFP) embryos injected with mutant mRNAs show perturbation of blood vessels in the caudal plexus and reduced ISVs development at 48 hpf. $(N=2)$

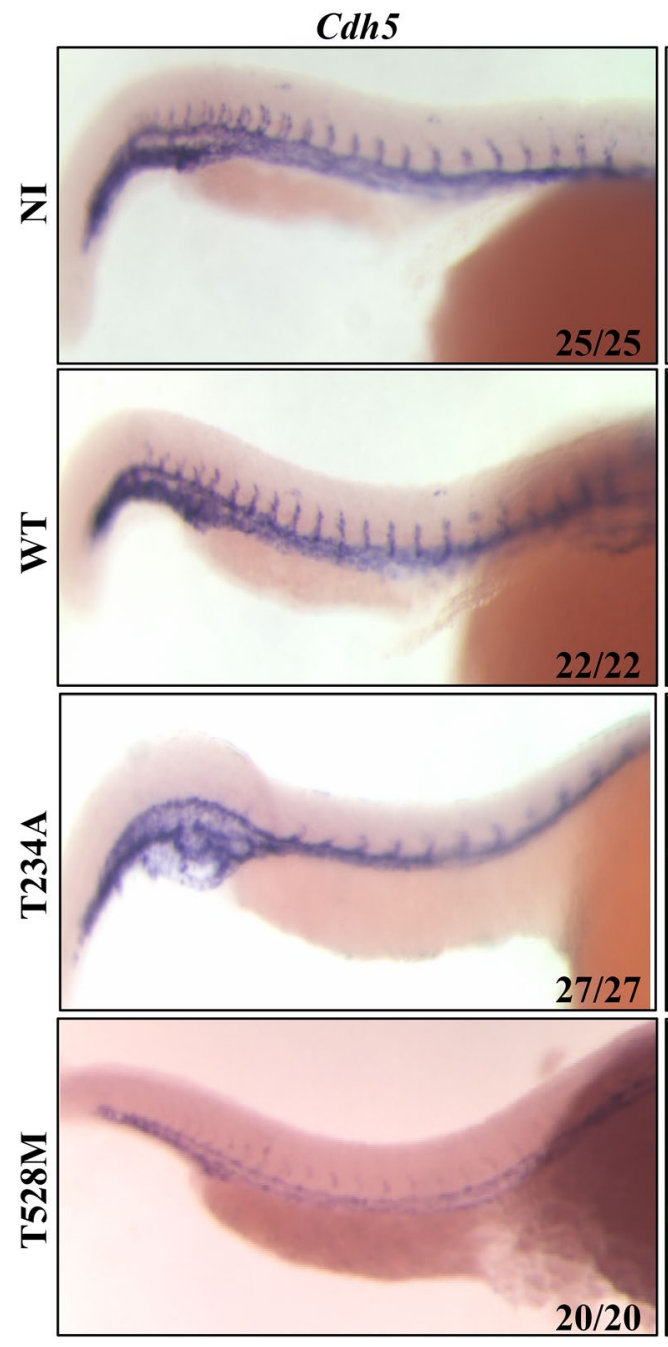

Tg(flila:EGFP)
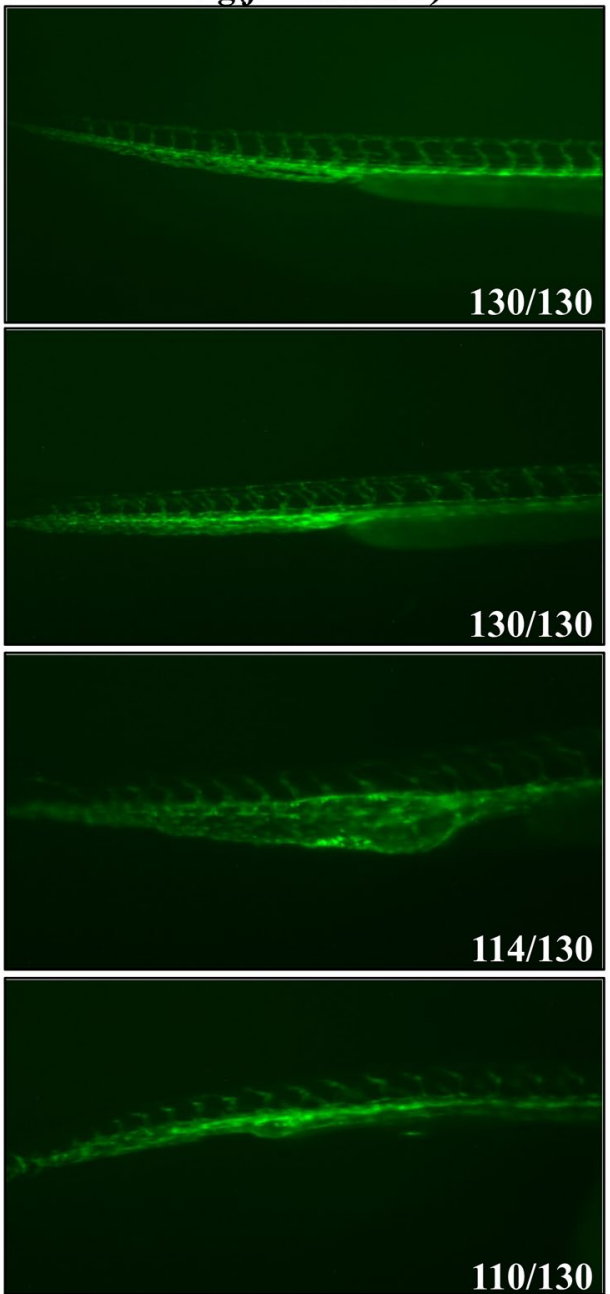

formation of irregular and supplementary vessels. Likewise, the expression of $c d h 5$ in embryos injected with T528M mutant mRNA was diminished to a drastically low level. Labeling in the ventral part of tail was completely missing, indicating the improper development of various blood vessels, which might have led to underdevelopment and curvature of the tail. In another experiment (Fig. 3, right panel), we used a reporter line $\operatorname{Tg}(f i l a: E G F P)$ which produces enhanced green fluorescent protein (EGFP) under the promoter of flila, a transcription factor highly expressed in endothelial cells and all hematopoietic tissues (Lawson and Weinstein 2002). Embryos were injected with WT and KON-Mt PANK2 mRNAs and analyzed at the fluorescent microscope at $48 \mathrm{hpf}$. Non-injected embryos and embryos injected with WT PANK2 mRNA were identical for distribution and intensity of EGFP signal. On the contrary, blood vessels formation was clearly improper at the caudal plexus in embryos injected with KON-Mt mRNAs, and particularly in embryos overexpressing the T234A PANK2. In both types of embryos, intersegmental vessels did not form properly and appeared still at sprouting stage when compared to control samples, with the parachordal lymphangioblasts being completely missing. The results are in line with the morphological observation described above and resembles to those obtained in embryos injected with a pank2-specific morpholino (Zizioli et al. 2016).

\section{Effect of Overexpression of WT and Mutant PANK2 mRNA on Neuronal Development}

While pank2 morphants showed perturbed cerebral structures, (Zizioli et al. 2016) brain development appeared to be normal in embryos overexpressing the mutant forms of PANK2. To explore this aspect in more details, we analyzed neuronal development by performing ISH for neurog 1 and neurod1, two key transcription factors for zebrafish neurogenesis (Mueller and Wullimann 2002). Non-injected embryos were taken as control for the experiment. No significant difference was observed in the expression of both genes in control or injected embryos (data not shown). 
These results were confirmed by using the transgenic line $\operatorname{Tg}$ (neurod1:EGFP), expressing EGFP under neurodl promoter. Distribution and intensity of fluorescence were similar in injected and non-injected control embryos (Online supplementary material, Figure S4). To explore further possible effects on neural cells, we stained embryos with AO, a fluorescent, cell-permeable dye that can be used to identify dead cells (Söderström 1977). We observed an increase in the number of cells positive for AO staining in the head of embryos overexpressing mutant PANK2 proteins at $48 \mathrm{hpf}$. The increase was more evident in embryos expressing KONMt forms (Online supplementary material, Figure S5), particularly in the eyes and the forebrain.

\section{CoA Prevents the Phenotype Associated With Mutant PANK2 Overexpression}

We previously showed that addition of $100 \mu \mathrm{M}$ CoA to fish water increased CoA levels in embryos (Khatri et al. 2016) and could prevent the perturbation of zebrafish embryo development induced by morpholinos targeting either PANK2 or COASY (Khatri et al. 2016; Zizioli et al. 2016). To verify whether the described phenotypic changes might be associated to perturbation of CoA homeostasis, we evaluated the rescue potential of CoA added to fish water. Embryos were injected with WT and mutant PANK2 mRNAs and allowed to grow at $28^{\circ} \mathrm{C}$ in fish water. Half of embryos were provided with exogenous $100 \mu \mathrm{M} \mathrm{CoA}$ in the fish water after $4 \mathrm{~h}$ of microinjection. At $48 \mathrm{hpf}$, all embryos were collected and analyzed (Fig. 4 and Online supplementary material, Figure S6). No difference was evident between control and WT mRNA-injected embryos, irrespective of CoA addition. Most embryos (G219V 139/150 and S471N 138/150) receiving KOFF-Mt mRNAs and CoA were hardly distinguishable from control ones: they developed normally with proper extension of the yolk; no hemorrhages were present, tail structure and size were appropriate, without any curvature. Most of the embryos (126/151) injected with the T234A mRNA showed a good recovery when supplied with exogenous $\mathrm{CoA}$ in fish water. The curved tail structure was completely rescued and all the embryos had a straight tail axis. The hemorrhage in caudal plexus was strongly reduced, blood flow to the lateral part of tail was also improved and most tissues at the tip of tail developed normally. Similar results were obtained with embryos overexpressing the T528M mutant PANK2: they showed (129/150) improvement in proper development of the tail when supplied with exogenous $\mathrm{CoA}$ in fish water. The axis of development was straight and hemorrhages markedly reduced. These experiments indicated that addition of exogenous CoA to fish water allowed a significant recovery of normal development in all embryos overexpressing mutant forms of human PANK2.

\section{Rescue of Aberrant Development by Supplementation of Vitamin B5}

We hypothesized that the overexpressed mutant proteins could block embryonic CoA production by limiting substrate availability for the endogenous Pank2. To challenge this hypothesis, we provided $50 \mu \mathrm{M}$ of vitamin B5 to the microinjected embryos at $4 \mathrm{hpf}$, in fish water (Fig. 4 and Online supplementary material, Figure S6). As previously documented (Zizioli et al. 2016), this dose had no effect on control embryos. Embryos injected with WT or mutant mRNAs showed the same features described above. On supplementation with vitamin B5, no changes were observed in embryos overexpressing WT PANK2, while most of the embryos overexpressing KON-Mt mRNAs (G219V 134/155 and S471N 139/151) were rescued and did not show hemorrhage in the tail. Similarly, the large majority of embryos injected with KOFF-Mt mRNAs (T234A 125/148 and T528M 133/155) developed normally, even though mild

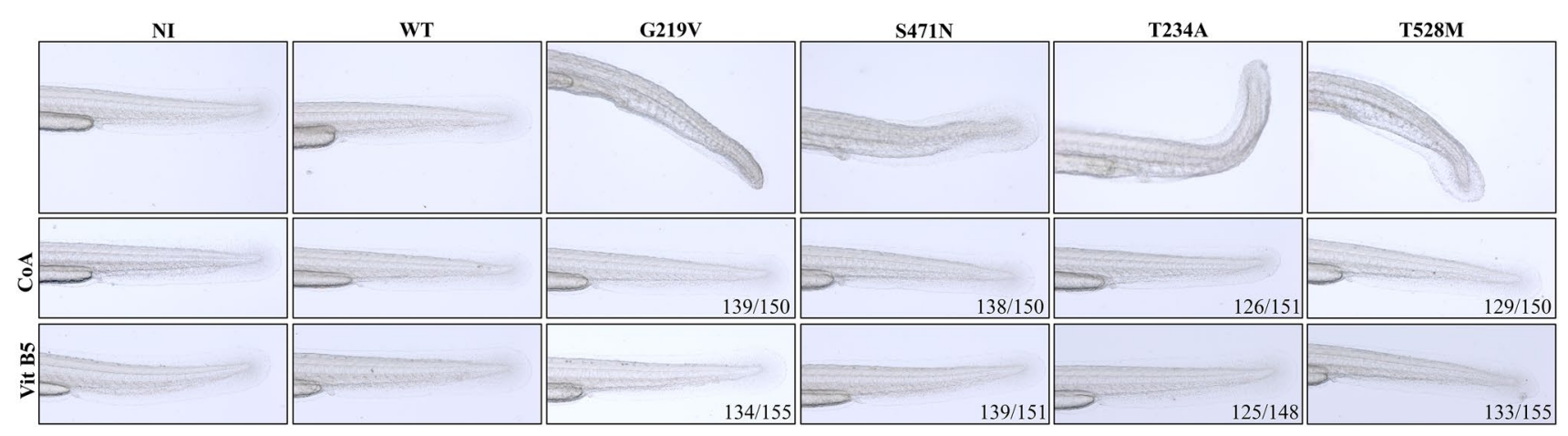

Fig. 4 Supplementation of CoA or vitamin B5 rescued the altered phenotype. Embryos were injected with different PANK2 mRNAs and $100 \mu \mathrm{M}$ CoA or $50 \mu \mathrm{M}$ vitamin B5 was added or not to fish water at 4 hpf. Both CoA and Vitamin B5 supplementation largely prevented the development of the morphological defects. Enlargements from lateral views of the tails are shown. Full images are shown in Online supplementary material, figure S6. Representative images from one experiment out of a total of 3 with similar results 
signs of blood vessels perturbation and blood cells accumulation in the tail were still present in some of them (T234A 58/125 and T528M 47/133). Altogether the rescuing strength of vitamin B5 appeared to be like that of CoA, suggesting a possible competition for the substrate between the overexpressed mutants and the endogenous Pank2. To confirm further the specific requirement for molecules involved in CoA biosynthesis and exclude a more general, trophic effect, we tested the effect of vitamin C. The addition of 25, 50 (not shown), and $100 \mu \mathrm{M}$ vitamin $\mathrm{C}$ at $4 \mathrm{hpf}$ did not affect the normal development of wild-type embryos. When added at the highest concentration to fish water of embryos injected with T234A mRNA, it did not prevent the appearance of tail hemorrhages and even induced a kink in the tail (Online supplementary material, Figure S7).

\section{Assessment of Locomotor Behavior}

Zebrafish embryos are a powerful model to investigate locomotor behavior and the development of the neural networks involved in it (McKeown et al. 2009). Starting from $17 \mathrm{hpf}$, they show spontaneous movement, and repeatedly perform slow, alternating tail coils. Rapid tail coils can be evoked by touch stimuli from $21 \mathrm{hpf}$, and finally spontaneous swimming can be observed from $27 \mathrm{hpf}$. We investigated the effects of PANK2 overexpression on locomotor activity by comparing first the spontaneous coiling contractions at $21 \mathrm{hpf}$, and then the touch-evoked swimming at $48 \mathrm{hpf}$. Embryos at $21 \mathrm{hpf}$ move 27-33 times/min (Basnet et al. 2017). The number of spontaneous movements of each injected and control embryo was counted for $1 \mathrm{~min}$. As shown in the box diagram (Fig. 5a), the number of spontaneous head and tail coil movements was significantly decreased at $21 \mathrm{hpf}$ in embryos injected with different mutant mRNAs, as compared to the embryos injected with WT PANK2 mRNA and non-injected controls (one-way ANOVA). Injection of mutant mRNAs caused a severe reduction in this kind of movements; their number was as low as 15 movements per minute for KOFF-Mt forms and even as low as 10 movements per minute for KON-Mt mRNAs.

Later we performed a touch-evoked movement test at $48 \mathrm{hpf}$ (Fig. 5b). The test was performed on groups of 40 embryos for each type of injected mRNAs and control. Each embryo was placed at the center of a motility wheel containing four concentric circles $(5,10,15$, and $20 \mathrm{~mm}$ diameter). The embryo was then touched gently in the tail with an embryo poker and the distance swam was recorded and categorized into five groups: $<5,5-10,10-15,15-20$, and $>20 \mathrm{~mm}$. All control and WT PANK2 mRNA-injected embryos could swim for more than $20 \mathrm{~mm}$. On the contrary, all embryos injected with mutant mRNAs covered a reduced distance and none of them was able to exit the $20 \mathrm{~mm}$ circle. Once again, embryos overexpressing KON-Mt forms showed a more severe phenotype than those with KOFF-Mt PANK2, with many embryos (T234A 50\% and T528M 60\%) gliding away for less than $10 \mathrm{~mm}$. Some embryos (G219V $10 \%$, S471N 16\%, T234A 40\% and T528M 45\%) showed diverse pattern, like around the circle movement as compared to embryos injected with WT mRNA.

A strong recovery of spontaneous coil movement was recorded in all embryos injected with different mutant mRNAs and supplemented with exogenous CoA. After
A

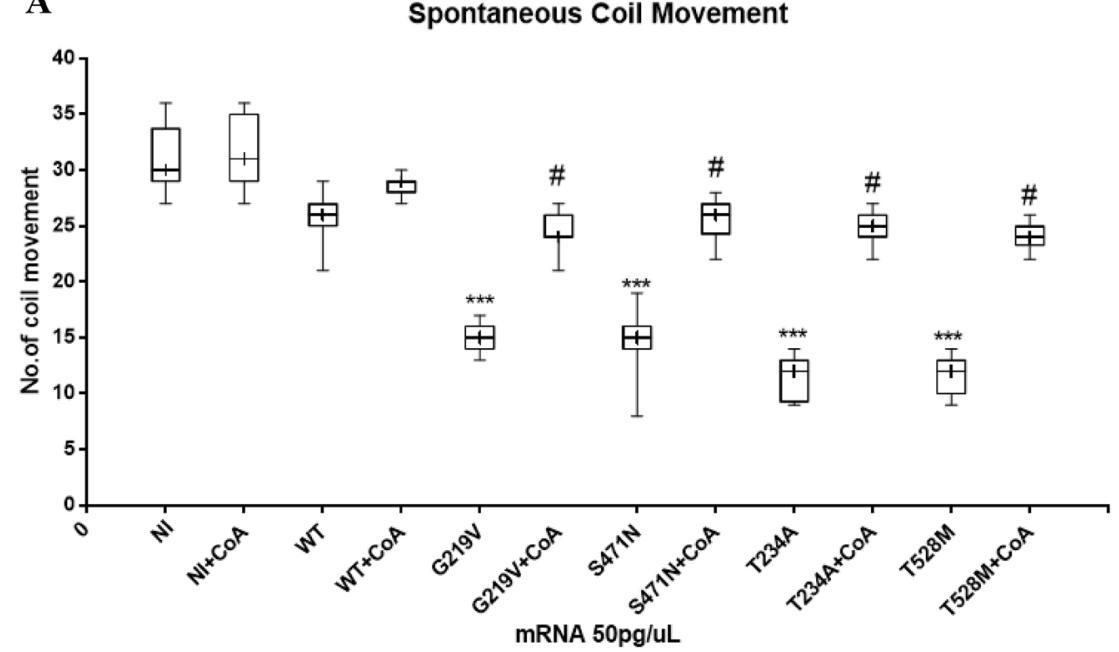

B

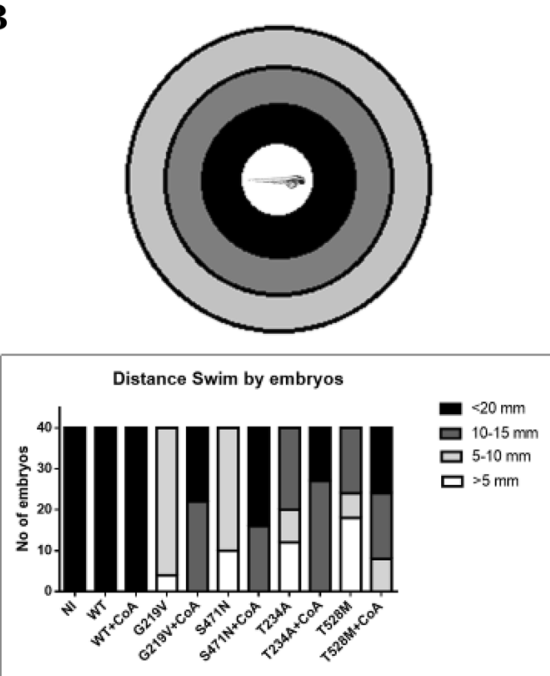

Fig. 5 Locomotor behavior analysis of embryos overexpressing human PANK2 mRNAs. a Embryos were injected with human WT and mutant PANK2 mRNAs and eventually provided with $100 \mu \mathrm{M}$ $\mathrm{CoA}$ in fish water at $4 \mathrm{hpf}$. Spontaneous coil movement was meas- ured at $21 \mathrm{hpf}$. NI non-injected embryos. b Touch-and-evoke test performed on microinjected embryos at $48 \mathrm{hpf}$. $Y$-axis shows the number of embryos that swam different distances. $(N=2)$ 
treatment with exogenous $100 \mu \mathrm{M} \mathrm{CoA}$, all embryos showed at least 22 coil movements, very similar to the number shown by WT PANK2-injected embryos ( $T$ Student test). A similar recovery was also observed in their gliding behavior; after treatment with CoA, half of the embryos went past $20 \mathrm{~mm}$ or they were able to glide at least a distance of $15 \mathrm{~mm}$.

\section{Discussion}

The molecular mechanisms of PKAN pathology are not completely understood yet (Levi and Finazzi 2014; Di Meo et al. 2018). As in all autosomal recessive disorders, variations in both alleles have been identified in most genetically diagnosed cases, supporting the hypothesis of a loss of function pathogenic mechanism. The biochemical consequence of the genetically determined PANK2 defects should be a shortage of CoA, more evident in cells with higher expression levels of the specific isoform and eventually with higher energy demand. Surprisingly, an in vitro biochemical analysis of many PKAN-associated mutations resulted in modest or no modification of the pantothenate kinase activity with no effects on protein stability or intracellular targeting, and sensitivity to feedback controls. An in vivo analysis conducted in the Drosophila $\mathrm{fbl}^{1}$ strain confirmed the results, with slight differences, thus reinforcing the question about the exact functional relationship between PANK2 enzyme and cellular CoA homeostasis. Furthermore, many mutations showed partial rescue efficiency when tested in vivo, in bacteria or Drosophila models (Wu et al. 2009; Hartig et al. 2006). We decided to investigate PANK2 mutant forms by overexpressing them in zebrafish embryos. We hypothesized that the limiting amount of vitamin B5 and CoA available in embryos and the low levels of expression of pank 2 could make this approach feasible also for loss of function mutants retaining a partial activity. We selected two mutations associated with a marked reduction of kinase activity (G219V and S471N-KOFF-Mt) and two (T234A and $\mathrm{T} 528 \mathrm{M}-\mathrm{KON}-\mathrm{Mt}$ ) with conservation of normal activity. Upon marked overexpression of mutant mRNAs, but not of WT mRNA, we observed a plethora of morphological and functional effects. They included reduction in trunk and tail thickness, curved tail, aberrant or absent tail yolk extension, hemorrhages at the tail plexus, reduced development of vascular structures and particularly of IVS. In addition, the locomotor activity development was perturbed as documented by the reduction in the spontaneous coiling movement first, and decreased swimming performance later. The observed phenotype is not an artefactual consequence of massive expression of an exogenous protein since same levels of WT protein had no effects on both morphology and behavior. Furthermore, the co-expression of WT and mutant protein at 1:1 ratio ( $25 \mathrm{pg}$ of mRNA each) resulted in normal embryo development, confirming the specificity of results. Interestingly all defects were prevented by the addition of CoA $100 \mu \mathrm{M}$ to fish water. Furthermore, also vitamin B5 was effective at preventing the development of the aberrant phenotype. The same vitamin did not rescue pank 2 morphants, and thus, it is unlikely that its effect is due to an increase of endogenous panks expression. Although we did not measure CoA levels in embryos, we can speculate that reduced CoA availability is the cause of the aberrant phenotype; most probably, high level of mutant protein competes for the substrate, vitamin B5, with the endogenous enzyme and precludes or dramatically reduces its normal activity. The results support the possibility that both KOFF and KON mutants can bind pantothenate. As a matter of fact, also K-OFF mutants retain a residual kinase activity in vitro. Differences in the severity of the observed phenotypes may relate to the affinity of specific mutants for pantothenate, with KON-Mt retaining a normal affinity and hence exerting strong competition for the substrate, and KOFF-Mt possibly having a reduced affinity and thereby a lower competitive capacity. Obviously more in depth analysis of the biochemical features of each mutant are required to verify all aspects of this hypothesis. Similarly, specific experiments are necessary to define the mechanism by which $\mathrm{KON}-\mathrm{mt}$ proteins affects CoA homeostasis in cells.

The high expression levels obtained in our system may limit the comparison with results obtained in the Drosophila $f b l^{1}$ model (Wu et al. 2009). It is interesting to observe that in the case of $f b l^{1}$ model, the starting point is represented by absent or very low level of PANK activity and overexpression of human PANK2 mutants partially restore it. This assay compares different mutant protein for residual catalytic activity. In our case, we start form $100 \%$ pank 2 activity and reduce it by overexpression of mutant, less active enzymes that suppress the endogenous enzymatic action. The final effect on CoA availability may be very similar in both conditions, even though obtained and looked at in a different way.

When we compared the phenotype induced by mutant mRNAs with that obtained by knocking down endogenous pank2 expression with specific morpholinos, we could observe significant similarities. In both cases, derangement of vascular structures development was evident with marked delay in IVS formation. Similarly, defects in the yolk extension at the tail were present in both type of embryos. For some aspect, and in particular for the presence of hemorrhages at the caudal plexus, the overexpression of mutant PANK2 forms recalled the phenotype of COASY morphants (Khatri et al. 2016). The expression of PANK2 mutants is obviously ubiquitous and not limited to tissues and cells carrying the endogenous enzyme. It is then possible that the competitive effect of mutant proteins is more generalized and eventually hits other endogenous panks. At the same time, some features observed in pank 2 morphants are very 
modest in embryos overexpressing mutant PANK2 forms. The edema and altered morphology of the brain were rarely observed upon mutant proteins overexpression as well as no significant changes in neural markers expression were evident. Yet, the positivity for AO staining was increased in embryos injected with mutant mRNAs, indicating a higher level of cell death. The absence of a full-blown neuronal phenotype could be related either to the temporal scale necessary to establish CoA deficiency or to the level of CoA deficiency. The downregulation of pank 2 by the morpholino may result in a more rapid or intense depletion of enzymatic activity and hence of CoA, thus affecting earlier and/ or less sensitive steps of embryonal differentiation, such as neurogenesis and neural differentiation. The overexpression of mutant forms of PANK2 may take a longer time to reduce CoA level or induce it only partially, thus affecting exclusively later and/or more sensitive stages of zebrafish development.

\section{Conclusion}

We have shown that PANK2 mutants induce an aberrant phenotype upon overexpression in zebrafish embryos. Curvature and reduced size of the trunk and tail, small or absent tail yolk extension, hemorrhages at the tail plexus and incomplete development of vascular structures and IVS were clearly present when KON-Mt and, to a lesser extent, KOFF-Mt mRNAs were microinjected. In addition, the locomotor activity development was affected. The effect is most probably related to the capacity of the overexpressed mutants to compete with the endogenous enzyme for the substrate vitamin B5, hence limiting CoA biosynthesis and levels in embryos. Indeed, the supplementation of vitamin B5 or CoA restored normal development. The data suggest that mutations in $P A N K 2$ are associated with perturbation in CoA availability, irrespective of the level of enzymatic activity retained by the mutant protein.

Acknowledgements We are grateful to dr. Paul Kotzbauer (Washington University School of Medicine, St. Louis, USA) for providing the recombinant plasmids of WT and mutant PANK2. The polyclonal antibody for human PANK1b was generously provided by dr. Suzanne Jackowski (St. Jude Children's Research Hospital, Memphis, USA). The research was supported by the "Health \& Wealth" funding from the University of Brescia (ZEBRACA project) to DF, Ex $60 \%$ funding from the University of Brescia to DF and DZ. AT is a PhD student form the Biomedical Sciences and Translational Medicine Program, University of Brescia.

Author Contributions DK planned and performed the experiments, analyzed the data, and corrected the manuscript; AT helped in planning and performing some experiments and revised the manuscript; GB and EM analyzed the data and revised the manuscript; DZ and DF conceived and planned the experiments, analyzed the data, prepared the figures, wrote and revised the manuscript.
Funding The work was supported by Health\&Wealth (project name ZEBRACA, DF) and ex60\% funds from the University of Brescia (DZ, $\mathrm{DF})$.

Availability of Data and Materials The datasets used and/or analyzed during the current study are available from the corresponding authors on reasonable request.

\section{Compliance with Ethical Standards}

Conflict of Interest Dr Deepak Khatri, dr Akansha Trivedi, dr Daniela Zizioli, Prof. Giuseppe Borsani, Prof. Eugenio Monti, and Prof. Dario Finazzi declare that they have no competing interest to disclose.

\section{References}

Alfonso-Pecchio, A., Garcia, M., Leonardi, R., \& Jackowski, S. (2012). Compartmentalization of mammalian pantothenate kinases. PLoS ONE, 7(11), e49509. https://doi.org/10.1371/journal.pone.00495 09.

Basnet, R. M., Guarienti, M., \& Memo, M. (2017). Zebrafish embryo as an in vivo model for behavioral and pharmacological characterization of methylxanthine drugs. International Journal of Molecular Science, 18(3), 596. https://doi.org/10.3390/ijms18030596.

Brunetti, D., Dusi, S., Giordano, C., Lamperti, C., Morbin, M., Fugnanesi, V., et al. (2014). Pantethine treatment is effective in recovering the disease phenotype induced by ketogenic diet in a pantothenate kinase-associated neurodegeneration mouse model. Brain, 137(Pt 1), 57-68. https://doi.org/10.1093/brain/awt325.

Brunetti, D., Dusi, S., Morbin, M., Uggetti, A., Moda, F., D’Amato, I., et al. (2012). Pantothenate kinase-associated neurodegeneration: Altered mitochondria membrane potential and defective respiration in Pank2 knock-out mouse model. Human Molecular Genetics, 21(24), 5294-5305. https://doi.org/10.1093/hmg/dds380.

Di Meo, I., Carecchio, M., \& Tiranti, V. (2018). Inborn errors of coenzyme A metabolism and neurodegeneration. Journal of Inherited Metabolic Disease, https://doi.org/10.1007/s10545-018-0193-0.

Dusi, S., Valletta, L., Haack, T. B., Tsuchiya, Y., Venco, P., Pasqualato, S., et al. (2014). Exome sequence reveals mutations in CoA synthase as a cause of neurodegeneration with brain iron accumulation. The American Journal of Human Genetics, 94(1), 11-22. https://doi.org/10.1016/j.ajhg.2013.11.008.

Gatta, L. B., Vitali, M., Verardi, R., Arosio, P., \& Finazzi, D. (2009). Inhibition of heme synthesis alters amyloid precursor protein processing. Journal of Neural Transmission, 116(1), 79-88. https:// doi.org/10.1007/s00702-008-0147-z.

Goody, M. F., Kelly, M. W., Reynolds, C. J., Khalil, A., Crawford, B. D., \& Henry, C. A. (2012). NAD+ biosynthesis ameliorates a zebrafish model of muscular dystrophy. PLoS Biology, 10(10), e1001409. https://doi.org/10.1371/journal.pbio.1001409.

Gregory, A., Polster, B. J., \& Hayflick, S. J. (2009). Clinical and genetic delineation of neurodegeneration with brain iron accumulation. Journal of Medical Genetics, 46(2), 73-80. https://doi. org/10.1136/jmg.2008.061929. pii]

Hartig, M. B., Hörtnagel, K., Garavaglia, B., Zorzi, G., Kmiec, T., Klopstock, T., et al. (2006). Genotypic and phenotypic spectrum of PANK2 mutations in patients with neurodegeneration with brain iron accumulation. Annals of Neurology, 59(2), 248-256. https://doi.org/10.1002/ana.20771.

Hayflick, S. J. (2003). Unraveling the Hallervorden-Spatz syndrome: Pantothenate kinase-associated neurodegeneration is the name. Current Opinion in Pediatrics, 15(6), 572-577. 
Khatri, D., Zizioli, D., Tiso, N., Facchinello, N., Vezzoli, S., Gianoncelli, A., et al. (2016). Down-regulation of coasy, the gene associated with NBIA-VI, reduces Bmp signaling, perturbs dorsoventral patterning and alters neuronal development in zebrafish. Scientific Reports, 6, 37660. https://doi.org/10.1038/srep37660.

Kimmel, C. B., Ballard, W. W., Kimmel, S. R., Ullmann, B., \& Schilling, T. F. (1995). Stages of embryonic development of the zebrafish. Developmental Dynamics, 203(3), 253-310.

Kotzbauer, P. T., Truax, A. C., Trojanowski, J. Q., \& Lee, V. M. (2005). Altered neuronal mitochondrial coenzyme A synthesis in neurodegeneration with brain iron accumulation caused by abnormal processing, stability, and catalytic activity of mutant pantothenate kinase 2. Journal of Neuroscience, 25(3), 689-698. https://doi. org/10.1523/JNEUROSCI.4265-04.2005.

Lawson, N. D., \& Weinstein, B. M. (2002). In vivo imaging of embryonic vascular development using transgenic zebrafish. Developmental Biology, 248(2), 307-318.

Leonardi, R., Rock, C. O., Jackowski, S., \& Zhang, Y. M. (2007a). Activation of human mitochondrial pantothenate kinase 2 by palmitoylcarnitine. Proceedings of the National Academy of Science USA, 104(5), 1494-1499. https://doi.org/10.1073/pnas.06076 21104.

Leonardi, R., Zhang, Y., Lykidis, A., Rock, C., \& Jackowski, S. (2007b). Localization and regulation of mouse pantothenate kinase 2. FEBS Letters, 581(24), 4639-4644. https://doi. org/10.1016/j.febslet.2007.08.056. pii].

Leonardi, R., Zhang, Y. M., Rock, C. O., \& Jackowski, S. (2005). Coenzyme A: Back in action. Progress in Lipid Research, 44(2-3), 125-153. https://doi.org/10.1016/j.plipres.2005.04.001.

Levi, S., \& Finazzi, D. (2014). Neurodegeneration with brain iron accumulation: Update on pathogenic mechanisms. Frontiers in Pharmacology, 5, 99. https://doi.org/10.3389/fphar.2014.00099.

McKeown, K. A., Downes, G. B., \& Hutson, L. D. (2009). Modular laboratory exercises to analyze the development of zebrafish motor behavior. Zebrafish, 6(2), 179-185. https://doi.org/10.1089/ zeb.2008.0564.

Montero-Balaguer, M., Swirsding, K., Orsenigo, F., Cotelli, F., Mione, M., \& Dejana, E. (2009). Stable vascular connections and remodeling require full expression of VE-cadherin in zebrafish embryos. PLOS ONE, 4(6), e5772. https://doi.org/10.1371/journ al.pone. 0005772 .

Mueller, T., \& Wullimann, M. F. (2002). Expression domains of neuroD (nrd) in the early postembryonic zebrafish brain. Brain Research Bulletin, 57(3-4), 377-379.

Nasevicius, A., \& Ekker, S. C. (2000). Effective targeted gene 'knockdown' in zebrafish. Nature Genetics, 26(2), 216-220. https://doi. org/10.1038/79951.

Orellana, D. I., Santambrogio, P., Rubio, A., Yekhlef, L., Cancellieri, C., Dusi, S., et al. (2016). Coenzyme A corrects pathological defects in human neurons of PANK2-associated neurodegeneration. EMBO Molecular Medicine, 8(10), 1197-1211. https://doi. org/10.15252/emmm.201606391.

Rana, A., Seinen, E., Siudeja, K., Muntendam, R., Srinivasan, B., van der Want, J. J., et al. (2010). Pantethine rescues a Drosophila model for pantothenate kinase-associated neurodegeneration. Proceedings of the National Academy of Science USA, 107(15), 6988-6993. https://doi.org/10.1073/pnas.0912105107.

Rock, C. O., Calder, R. B., Karim, M. A., \& Jackowski, S. (2000). Pantothenate kinase regulation of the intracellular concentration of coenzyme A. Journal of Biological Chemistry, 275(2), $1377-1383$

Siudeja, K., Srinivasan, B., Xu, L., Rana, A., de Jong, J., Nollen, E. A., et al. (2011). Impaired Coenzyme A metabolism affects histone and tubulin acetylation in Drosophila and human cell models of pantothenate kinase associated neurodegeneration. EMBO Molecular Medicine, 3(12), 755-766. https://doi.org/10.1002/ emmm.201100180.

Söderström, K.-O., Parvinen, L.-M., \& Parvinen, M. (1977). Early detection of cell damage by supravital acridine orange staining. Experientia, 33(2), 265-266.

Srinivasan, B., Baratashvili, M., van der Zwaag, M., Kanon, B., Colombelli, C., Lambrechts, R. A., et al. (2015). Extracellular 4'-phosphopantetheine is a source for intracellular coenzyme A synthesis. Nature Chemical Biology, 11(10), 784-792. https://doi. org/10.1038/nchembio.1906.

Thisse, C., \& Thisse, B. (2008). High-resolution in situ hybridization to whole-mount zebrafish embryos. Nature Protocols, 3(1), 59-69. https://doi.org/10.1038/nprot.2007.514.

Wu, Z., Li, C., Lv, S., \& Zhou, B. (2009). Pantothenate kinase-associated neurodegeneration: insights from a Drosophila model. Human Molecular Genetics, 18(19), 3659-3672. https://doi. org/10.1093/hmg/ddp314.

Zhang, Y. M., Chohnan, S., Virga, K. G., Stevens, R. D., Ilkayeva, O. R., Wenner, B. R., et al. (2007). Chemical knockout of pantothenate kinase reveals the metabolic and genetic program responsible for hepatic coenzyme A homeostasis. Chemistry Biology, 14(3), 291-302. https://doi.org/10.1016/j.chembiol.2007.01.013.

Zhang, Y. M., Rock, C. O., \& Jackowski, S. (2006). Biochemical properties of human pantothenate kinase 2 isoforms and mutations linked to pantothenate kinase-associated neurodegeneration. Journal of Biological Chemistry, 281(1), 107-114. https://doi. org/10.1074/jbc.M508825200.

Zizioli, D., Tiso, N., Guglielmi, A., Saraceno, C., Busolin, G., Giuliani, R., et al. (2016). Knock-down of pantothenate kinase 2 severely affects the development of the nervous and vascular system in zebrafish, providing new insights into PKAN disease. Neurobiology of Disease, 85, 35-48. https://doi.org/10.1016/j. nbd.2015.10.010. 